\title{
Veterans Health Administration
}

National Cancer Institute

\section{Source}

National Cancer Institute. Veterans Health Administration. NCI Thesaurus. Code C157363.

The largest integ rated health care system in the United States, providing health care services to over 9 million veterans enrolled in the VA health care program. The VHA manages 1,250 health care facilities, including 172 VA Medical Centers and 1,069 outpatient sites and clinics. 